See discussions, stats, and author profiles for this publication at: https://www.researchgate.net/publication/329311628

\title{
Normal lubrication force between spherical particles immersed in a shear- thickening fluid
}

Article in Physics of Fluids · December 2018

DOI: $10.1063 / 1.5054067$

CITATIONS

0

3 authors, including:

Adolfo Vázquez-Quesada

Universidad Autónoma de Madrid

22 PUBLICATIONS 233 CITATIONS

SEE PROFILE

Some of the authors of this publication are also working on these related projects:

Project I am working on applications of the Master kinetic equation (2014-2015) View project
READS

45

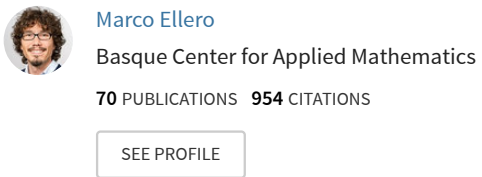




\title{
Normal lubrication force between spherical particles immersed in a shear-thickening fluid
}

\author{
A. Vázquez-Quesada, ${ }^{1, a)}$ N. J. Wagner, ${ }^{2, \text { b) }}$ and M. Ellero ${ }^{3,4,5, c)}$ \\ 1) Department of Theoretical Condensed Matter Physics, \\ Universidad Autónoma de Madrid, 28049 Madrid, Spain \\ 2) Department of Chemical and Biomolecular Engineering, University of Delaware, \\ 150 Academy Street, Colburn Laboratory, Newark, DE 19716, \\ $U S A$. \\ 3) Basque Center for Applied Mathematics (BCAM), \\ Alameda de Mazarredo 14, 48400 Bilbao, Spain \\ 4) IKERBASQUE, Basque Foundation for Science, \\ Calle de María Díaz de Haro 3, 48013 Bilbao, Spain \\ 5) Zienkiewicz Centre for Computational Engineering (ZCCE), Swansea University, \\ Bay Campus, Swansea SA1 8EN, United Kingdom.
}

In this work, the inverse bi-viscous model [Physics of Fluids 29, 103104 (2017)] is used to describe a shear-thickening fluid. An analytical velocity profile in a planar Poiseuille flow is utilized to calculate an approximate solution to the generalized lubrication force between two close spheres interacting hydrodynamically in such a medium. This approximate analytical expression is compared to the exact numerical solution. The flow topology of the shear-thickening transition within the interparticle gap is also shown and discussed in relation to the behaviour of the lubrication force. The present result can allow in the future to perform numerical simulations of dense particle suspensions immersed in a shear-thickening matrix based on an effective lubrication force acting between pairwise interacting particles. This model may find additional value in representing experimental systems consisting of suspensions in shear thickening media, polymer coated suspensions, and industrial systems such as concrete.

\footnotetext{
a) Electronic mail: adolfo.vazquez@uam.es

b) Electronic mail: wagnernj@udel.edu

c)Electronic mail: mellero@bcamath.org
} 


\section{INTRODUCTION}

Particulate suspensions are ubiquitous in nature and industrial applications, and the understanding of their flow properties represents both a challenging academic and technical problem. From a computational point of view, the dilute and semi-dilute regimes are generally amenable of direct numerical simulations where one resolves precisely the flow of the suspending phase and the rigid body dynamics of the suspended particles is coupled to the surrounding fluid solution via suitable fluid/structure-interaction schemes (e.g. detailed force balance, distributed Lagrange multiplier, force coupling method or immersed boundary methods $)^{1}$. These approaches have been successfully applied to suspensions with Newtonian and non-Newtonian matrices using finite volumes/elements techniques ${ }^{2-5}$ and Lagrangian methods ${ }^{6-9}$.

In very dense systems however, particles under flow can get very close to each other (i.e. the average interparticle separation is much smaller than the particle radius) entering the socalled lubrication regime. In this case long-range hydrodynamic interactions are screened out and one can restrict the multi-body problem to a set of solid particles interacting pairwisely via short-range dissipative (i.e. lubrication) forces. This approach allows to bypass the need to resolve flow features at very small scales - i.e. in the vanishing gaps between nearly touching objects - which can be numerically very time consuming. On the other hand, this 'simplification' introduces novel challenges of both, numerical and modelling nature. From a computational perspective, analytical solutions for the lubrication drag resulting from the squeezing flow between close spheres is diverging for vanishing interparticle gaps and it requires specific implicit techniques for an accurate and stable integration ${ }^{10,11}$. With regard to the modelling issue, although the problem of nearly touching lubricated spheres in a simple Newtonian solvent has been widely studied, there are scarce analytical works focusing on the corresponding problem with non-Newtonian suspending media ${ }^{12-14}$. At the same time, dense particulate systems interacting with complex non-Newtonian media are very common in practical applications and it is therefore of paramount importance to address the problem of generalized lubrication interactions in complex liquids. For a review, see Chapter 10 of Ref. $^{15}$ and $^{16-18}$.

$\operatorname{In}^{19}$ we have studied the problem of lubrication between two spheres in a shear-thinning medium modelled by the so-called bi-viscosity model. This was originally introduced in ${ }^{20}$ 
to model viscoplastic fluids with a pseudo-yield stress. Recently the model has been also used to incorporate shear-rate dependent slippage effects ${ }^{21}$ on effective lubrication between particles.

In this work, we consider the alternative case of a suspending non-Newtonian liquid exhibiting discontinuous shear-thickening (DST) rheological response and derive the resulting analytical expression for the interparticle lubrication force between two solid spheres suspended in such a medium. DST is a general phenomenon in several complex liquids and refers to a significant increase of liquid viscosity when large shear-rates or stresses are applied $^{22}$. It happens typically at very large shear rates and has profound implications for several processing conditions as it limits transport of paints, inks and other concentrated dispersions in spraying, coating and pumping operations but, when properly controlled, can be also used to engineer novel materials as selective dampers and shock absorbers ${ }^{23,24}$.

Specifically to the system considered here, an analytical pairwise lubrication force between particles immersed in a DST matrix can be used to perform efficient particulate flow simulations of the kind of complex suspension studied experimentally in ${ }^{25}$, using minimal pairwise lubrication models, i.e. without the need to resolve the entire flow around the particles.

In this work we consider two spheres immersed in a shear-thickening fluid and we aim at the analytical derivation of the resulting complex lubrication force acting on them. As we said before, the shear-thickening fluid is modelled as an inverse biviscous model with viscosity defined $\operatorname{as}^{26}$

$$
\eta(\dot{\gamma})=\left\{\begin{array}{cc}
\eta_{0}, & \text { if } \dot{\gamma} \leq \dot{\gamma}_{c}-\delta \dot{\gamma} \\
m \dot{\gamma}+n, & \text { if } \dot{\gamma}_{c}-\delta \dot{\gamma}<\dot{\gamma} \leq \dot{\gamma}_{c}+\delta \dot{\gamma} \\
\eta_{1}, & \text { if } \dot{\gamma} \geq \dot{\gamma}_{c}+\delta \dot{\gamma}
\end{array}\right.
$$

where $\eta_{1}>\eta_{0}$ and $m$ and $n$ are constants ensuring the continuity of the viscosity function and momentum balance. As discussed in Ref. ${ }^{26}$, a truly DST model is obtained as a limit of $\delta \dot{\gamma} \rightarrow 0$ in the regularized model presented above and it captures the essential physics of sudden increase in flow resistance of DST fluids, but modelling is limited to a generalized Newtonian framework. In this paper, we follow the route proposed in ${ }^{19}$ to derive the overall 


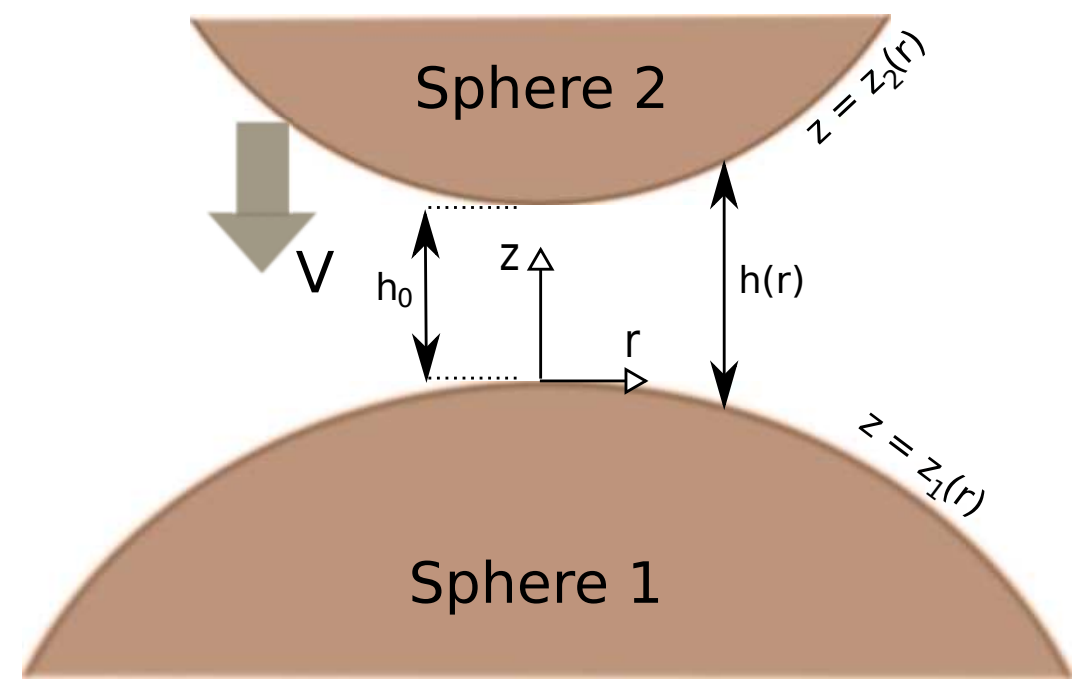

FIG. 1. Scheme of the problem of lubrication between two spheres.

lubrication force acting on each sphere as a result of the squeezing flow in/out of the gap for departing/approaching particles. In order to derive a closed-form analytical expression, we use the analytical solution for the pressure-driven flow of a DST fluid in a channel flow and use it to model the radial squeezing flow component ${ }^{26}$.

The structure of the paper is the following one: in Sec. 2, as a corollary the preliminary result for the flow of a inverse bi-viscous fluid driven by a constant pressure gradient between two parallel plates is discussed. This result is used in Sec. 3, where the calculation of the normal lubrication force between two spheres is presented. We consider the case of noncolloidal spherical particles suspended in a DST-liquids in Sec. 4 where the effect of relative particle velocity, viscosity ratio $\eta_{1} / \eta_{0}$ as well as choice of shear rate $\dot{\gamma}_{c}$ are explored. The local topology of the regions with different viscosities is also analyzed in Sec. 4. Finally conclusions are provided in Sec. 5 .

\section{CALCULATION OF THE NORMAL LUBRICATION FORCE}

\section{A. Definition of the different regions}

Let us consider the squeezing flow between two very close spheres of radius $a_{1}$ and $a_{2}$. The normal relative velocity between spheres is $V$. The system of reference and geometry of the problem is summarized in Fig. 1. 
If we consider only small gaps between spheres, their surfaces $z_{1}(r)$ and $z_{2}(r)$ can be approximated by paraboloids. Thus, the distance between them can be defined as $h(r)=$ $h_{0}\left(1+\frac{r^{2}}{2 a h_{0}}\right)$, where $\frac{1}{a}=\frac{1}{a_{1}}+\frac{1}{a_{2}}$ and $h_{0}$ is the distance between surfaces at $r=0^{27}$.

As in the lubrication force calculation between two spheres in a Newtonian fluid ${ }^{27}$, we can consider that the squeezing fluid is moving uni-directionally along the $r$ coordinate if the velocity $V$ is sufficiently small. The result obtained $i^{26}$ for the planar flow of an inverse bi-viscous shear-thickening fluid, with the rheology given by Eq. (1), under the influence of a constant pressure gradient can be then used here. For a given coordinate $r$, different velocity profiles can be distinguished depending on the value of the following lengths:

$$
\begin{aligned}
& z_{c}(r)=-\frac{\eta_{0} \dot{\gamma}_{c}}{\partial p / \partial r(r)} \\
& z_{c}^{\prime}(r)=-\frac{\eta_{1} \dot{\gamma}_{c}}{\partial p / \partial r(r)}=\frac{\eta_{1}}{\eta_{0}} z_{c}(r)
\end{aligned}
$$

where the pressure gradient $\partial p / \partial r(r)$ has been considered negative (squeezing flow) and its formal expression still needs to be determined. For the sake of completeness we briefly explain the effect of $z_{c}(r)$ and $z_{c}^{\prime}(r)$ on the velocity profiles. For a further understanding of the reader is referred to Ref. ${ }^{26}$. Three different regimes can be distinguished: if $z_{c} \geq h(r) / 2$, the critical shear rate $\dot{\gamma}_{c}$ is not reached at any $z$ coordinate, and the solution at coordinate $r$ reduces to the standard Poiseuille flow for a mono-viscous fluid of viscosity $\eta_{0}$ (regime $I$ ). If $z_{c}<h(r) / 2$ and $z_{c}^{\prime} \geq h(r) / 2$ the fluid at the coordinate $r$ is in the regime $I I$, where a limitation in the shear rate to $\dot{\gamma}_{c}$ shows up in the closest regions of the gap to the wall ${ }^{26}$. Such a limitation comes from the fact that when the critical shear rate $\dot{\gamma}_{c}$ is reached, the viscosity is increased, and the shear rate reduces below $\dot{\gamma}_{c}$. In the practice, this means that in those regions in the limit of $\delta \dot{\gamma} \rightarrow 0$ the shear rate will be equal to $\dot{\gamma}_{c}$. Finally, if $z_{c}^{\prime}<h(r) / 2$ the regions of limited shear rate get closer to the center, displaced by a region of viscosity $\eta_{1}$ (regime $\left.I I I\right)$. The velocity profile can be written then as follows:

$$
u(r, z)= \begin{cases}\left.u(r, z)\right|_{I} & , \text { if } r \in \mathcal{R}_{I} \\ \left.u(r, z)\right|_{I I} & , \text { if } r \in \mathcal{R}_{I I} \\ \left.u(r, z)\right|_{I I I} & , \text { if } r \in \mathcal{R}_{I I I}\end{cases}
$$

where $\mathcal{R}_{I}, \mathcal{R}_{I I}$ and $\mathcal{R}_{I I I}$ are, respectively, the regions of the space where the fluid is in the regimes $I, I I$ or $I I I$ respectively. The explicit expressions of the velocity profiles have been 
calculated in section II C from Ref. ${ }^{26}$ and read

$$
\begin{aligned}
&\left.u(r, z)\right|_{I}= \frac{1}{2 \eta_{0}} \frac{\partial p}{\partial r}\left((z-c)^{2}-\frac{h^{2}}{4}\right) \\
&\left.u(r, z)\right|_{I I}=\left\{\begin{array}{cc}
\dot{\gamma}_{c}\left(z-c+\frac{h}{2}\right) & \text { if } z<c-z_{c} \\
\frac{1}{2 \eta_{0}} \frac{\partial p}{\partial r}\left((z-c)^{2}+z_{c}\left(z_{c}-h\right)\right) & \text { if }|z-c| \leq z_{c} \\
-\dot{\gamma}_{c}\left(z-c-\frac{h}{2}\right) & \text { if } z>c+z_{c}
\end{array}\right. \\
&\left.u(r, z)\right|_{I I I}=\left\{\begin{array}{cc}
\frac{1}{2 \eta_{0}} \frac{\partial p}{\partial r}\left((z-c)^{2}+z_{c}\left(z_{c}-2 z_{c}^{\prime}\right)\right)+\frac{1}{2 \eta_{1}} \frac{\partial p}{\partial r}\left(z_{c}^{\prime 2}-\frac{h^{2}}{4}\right) & \text { if }|z-c| \leq z_{c} \\
\dot{\gamma}_{c}\left(z-\left(c-z_{c}^{\prime}\right)\right)+\frac{1}{2 \eta_{1}} \frac{\partial p}{\partial r}\left(z_{c}^{\prime 2}-\frac{h^{2}}{4}\right) & \text { if } c-z_{c}^{\prime} \leq z<c-z_{c} \\
-\dot{\gamma}_{c}\left(z-\left(c+z_{c}^{\prime}\right)\right)+\frac{1}{2 \eta_{1}} \frac{\partial p}{\partial r}\left(z_{c}^{\prime 2}-\frac{h^{2}}{4}\right) & \text { if } c+z_{c}<z \leq c+z_{c}^{\prime} \\
\frac{1}{2 \eta_{1}} \frac{\partial p}{\partial r}\left((z-c)^{2}-\frac{h^{2}}{4}\right) & \text { if }|z-c|>z_{c}^{\prime}
\end{array}\right.
\end{aligned}
$$

where $c(r)=\left(z_{1}(r)+z_{2}(r)\right) / 2$ defines the center position of the channel. The use of $I$, II and III subscripts will be used along this work with the meaning defined in the above equation. Note that the quantities $z_{1}, z_{2}, c, h, z_{c}, z_{c}^{\prime}$ and $\partial p / \partial r$ depend on the $r$ coordinate. For the sake of clarity such a dependency has not been explicitly written. The DST velocity profiles reported in Eq. (4) approximate very well the ones corresponding to the regularized DST version (1) when $\delta \dot{\gamma} / \dot{\gamma}_{c} \ll 1^{26}$. Note also that thanks to the assumption that $h_{0} \ll a$ the squeezing flow is unidirectional and radial.

For a Newtonian fluid, the maximum shear rate $\dot{\gamma}_{s}$ for a given position $r$, which is located at the surfaces of the spheres, is given by ${ }^{19}$

$$
\dot{\gamma}_{s}(r)=-\left.\frac{1}{\eta_{0}} \frac{\partial p}{\partial r}(r)\right|_{\text {newt }} \frac{h(r)}{2}=\frac{3 r V}{h^{2}(r)}
$$

This function, which has been drawn in Fig. 2, is characterized by a maximum value $\dot{\gamma}_{s}^{\max }$ at the position

$$
r_{\max }=\sqrt{\frac{2}{3} a h_{0}}
$$

whose value is given by

$$
\dot{\gamma}_{s}^{\max } \equiv \dot{\gamma}_{s}\left(r_{\max }\right)=\frac{9}{8} \frac{V}{h_{0}} \sqrt{\frac{3}{2} \frac{a}{h_{0}}}
$$

Therefore, the condition to obtain a non-Newtonian behavior at some coordinate $r$ is generally defined as

$$
\dot{\gamma}_{c}<\dot{\gamma}_{s}^{\max }
$$




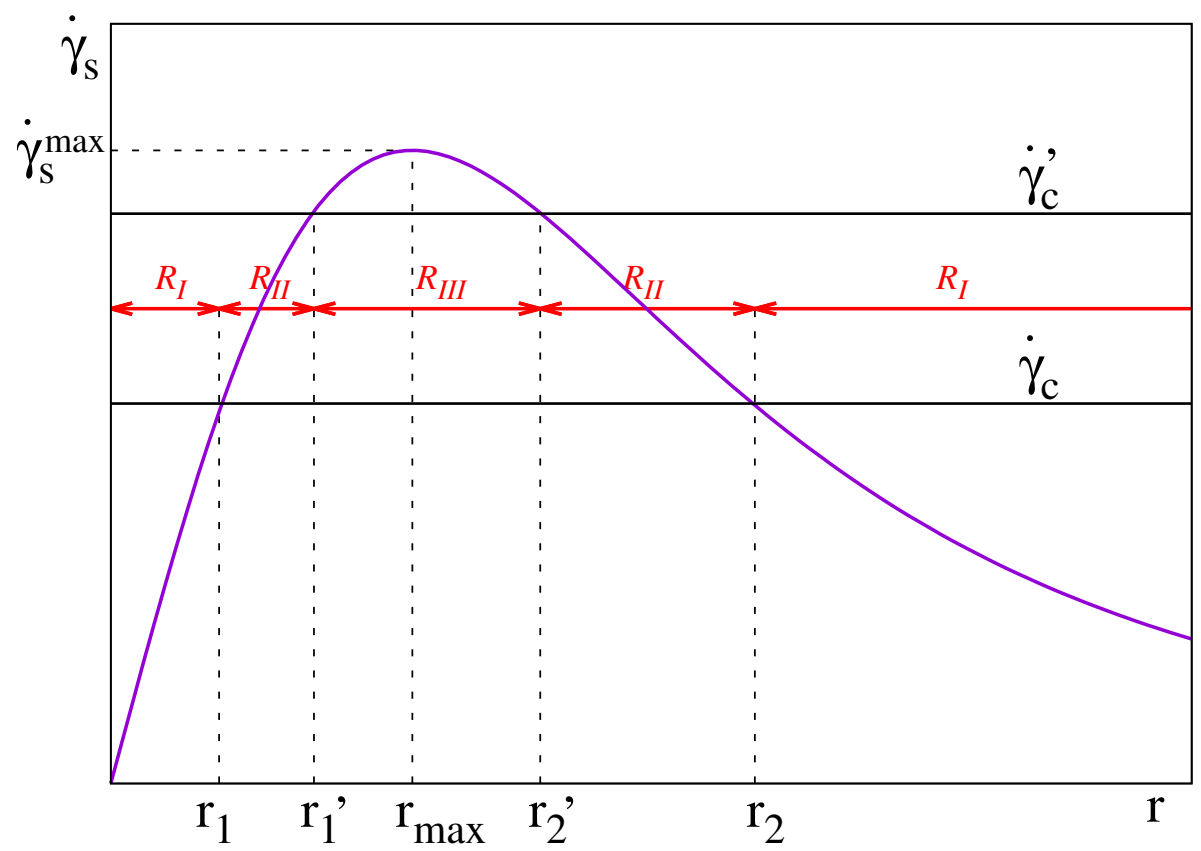

FIG. 2. The maximum shear rate $\dot{\gamma}_{s}(r)$ depending on $r$ of a Newtonian fluid. The pressure gradient of the fluid will be different than in the Newtonian case in the region $r_{1}<r<r_{2}$ (regions $\mathcal{R}_{I I}$ and $\left.\mathcal{R}_{I I I}\right)$.

which ensures that there will be some $r$ coordinate in the regime $I I$. There will be another critical shear rate $\dot{\gamma}_{c}^{\prime}>\dot{\gamma}_{c}$, that will be used to determine the transition of the biviscous liquid to have a non-null $\mathcal{R}_{I I I}$ region. The $\dot{\gamma}_{c}^{\prime}$ condition will be as follows

$$
\dot{\gamma}_{c}^{\prime}<\dot{\gamma}_{s}^{\max }
$$

Note that we are using the maximum shear rate of a Newtonian fluid as the reference to compare, although is not the real maximum shear rate in the regions $\mathcal{R}_{I I}$ and $\mathcal{R}_{I I I}$. This is convenient given that $\dot{\gamma}_{s}^{\max }$ does not depend on the viscosity, but it also means that $\dot{\gamma}_{c}^{\prime}$ is not representing the real limiting shear rate of the bi-viscous fluid to have a non-null region $\mathcal{R}_{I I I}$. Instead it represents the maximum shear rate that a Newtonian fluid would have under the same conditions of the bi-viscous one, when it goes through the transition. The explicit relationship between $\dot{\gamma}_{c}$ and $\dot{\gamma}_{c}^{\prime}$ will be calculated later, at section II D.

Three possibilities can be described related to these critical shear rates; if $\dot{\gamma}_{c} \geq \dot{\gamma}_{s}^{\max }$ the fluid behaves as Newtonian in every $r$ coordinate, so $r \in \mathcal{R}_{I}, \forall r$. If $\dot{\gamma}_{c}<\dot{\gamma}_{s}^{\max }$ and $\dot{\gamma}_{c}^{\prime} \geq \dot{\gamma}_{s}^{\max }$ 
two different regions appear, which can be defined as

$$
\begin{aligned}
& r \in \mathcal{R}_{I} \text { if } r \leq r_{1} \text { or } r \geq r_{2} \\
& r \in \mathcal{R}_{I I} \text { if } r_{1}<r<r_{2}
\end{aligned}
$$

Finally, if $\dot{\gamma}_{c}<\dot{\gamma}_{s}^{\max }$ and $\dot{\gamma}_{c}^{\prime}<\dot{\gamma}_{s}^{\max }$ three different regions can be distinguished

$$
\begin{aligned}
r \in \mathcal{R}_{I} & \text { if } r \leq r_{1} \text { or } r \geq r_{2} \\
r \in \mathcal{R}_{I I} & \text { if } r_{1}<r \leq r_{1}^{\prime} \text { or } r_{2}^{\prime} \leq r<r_{2} \\
r \in \mathcal{R}_{I I I} & \text { if } r_{1}^{\prime}<r<r_{2}^{\prime}
\end{aligned}
$$

The latter case is the one drawn at Fig. 2.

\section{B. Calculation of $r_{1}, r_{2}, r_{1}^{\prime}$ and $r_{2}^{\prime}$}

Calculation of $r_{1}$ and $r_{2}$ is made in a similar way to what done in Ref. ${ }^{19}$. Such a coordinates can be found by calculating the distances $r$ where $\dot{\gamma}_{s}(r)$ is equal to $\dot{\gamma}_{c}$ (see Fig. 2), which leads us to the next equation

$$
\left(\frac{r}{r_{\max }}\right)^{4}+6\left(\frac{r}{r_{\max }}\right)^{2}-16\left(\frac{\dot{\gamma}_{s}^{\max }}{\dot{\gamma}_{c}}\right)\left(\frac{r}{r_{\max }}\right)+9=0
$$

where $r_{\max }$ was defined in (6). Eq. (11) is a quartic equation which has unique real solutions given by

$$
\begin{aligned}
& r_{1}=r_{\max }\left[\mathcal{Q}-\sqrt{-3-\mathcal{Q}^{2}+4\left(\frac{\dot{\gamma}_{s}^{\max }}{\dot{\gamma}_{c}}\right) \frac{1}{\mathcal{Q}}}\right] \\
& r_{2}=r_{\max }\left[\mathcal{Q}+\sqrt{-3-\mathcal{Q}^{2}+4\left(\frac{\dot{\gamma}_{s}^{\max }}{\dot{\gamma}_{c}}\right) \frac{1}{\mathcal{Q}}}\right]
\end{aligned}
$$

where

$$
\begin{aligned}
& \mathcal{Q}=\sqrt{-1+\frac{1}{\mathcal{P}}+\mathcal{P}} \\
& \mathcal{P}=\sqrt[3]{-1+2\left(\frac{\dot{\gamma}_{s}^{\text {max }}}{\dot{\gamma}_{c}}\right)^{2}+2\left(\frac{\dot{\gamma}_{s}^{\text {max }}}{\dot{\gamma}_{c}}\right) \sqrt{\left(\frac{\dot{\gamma}_{s}^{\text {max }}}{\dot{\gamma}_{c}}\right)^{2}-1}}
\end{aligned}
$$


Expressions for $r_{1}^{\prime}$ and $r_{2}^{\prime}$ can be obtained by replacing $\dot{\gamma}_{c}^{\prime}$ for $\dot{\gamma}_{c}$ in $r_{1}$ and $r_{2}$ expressions, leading to

$$
\begin{aligned}
& r_{1}^{\prime}=r_{\max }\left[\mathcal{Q}^{\prime}-\sqrt{-3-\mathcal{Q}^{\prime 2}+4\left(\frac{\dot{\gamma}_{s}^{\max }}{\dot{\gamma}_{c}^{\prime}}\right) \frac{1}{\mathcal{Q}^{\prime}}}\right] \\
& r_{2}^{\prime}=r_{\max }\left[\mathcal{Q}^{\prime}+\sqrt{-3-\mathcal{Q}^{\prime 2}+4\left(\frac{\dot{\gamma}_{s}^{\max }}{\dot{\gamma}_{c}^{\prime}}\right) \frac{1}{\mathcal{Q}^{\prime}}}\right]
\end{aligned}
$$

being

$$
\begin{aligned}
& \mathcal{Q}^{\prime}=\sqrt{-1+\frac{1}{\mathcal{P}^{\prime}}+\mathcal{P}^{\prime}} \\
& \mathcal{P}^{\prime}=\sqrt[3]{-1+2\left(\frac{\dot{\gamma}_{s}^{\text {max }}}{\dot{\gamma}_{c}^{\prime}}\right)^{2}+2\left(\frac{\dot{\gamma}_{s}^{\text {max }}}{\dot{\gamma}_{c}^{\prime}}\right) \sqrt{\left(\frac{\dot{\gamma}_{s}^{\text {max }}}{\dot{\gamma}_{c}^{\prime}}\right)^{2}-1}}
\end{aligned}
$$

\section{Calculation of the minimum gaps to have regions in the regimes $I I$ and $I I I$}

The calculation of the minimum gap $h_{0}^{\lim }$ to have any non-null region in the regime $I I$ can be done similarly to the way outlined in Ref. ${ }^{19}$ by equating $\dot{\gamma}_{s}^{\max }=\dot{\gamma}_{c}$ at Eq. (7), and it yields

$$
h_{0}^{\lim }=\frac{3}{4} \sqrt[3]{\frac{9}{2} \frac{V^{2} a}{\dot{\gamma}_{c}^{2}}}
$$

In the same way, by doing $\dot{\gamma}_{s}^{\max }=\dot{\gamma}_{c}^{\prime}$ at Eq. (7), one can calculate the minimum gap between spheres $h_{0}^{\text {llim }}$ to observe the regime III in some $r$ coordinate. It reads

$$
h_{0}^{\prime l i m}=h_{0}^{\lim } \sqrt[3]{\frac{4}{\left(3-\left(\eta_{0} / \eta_{1}\right)^{2}\right)^{2}}}
$$

\section{Pressure gradient calculation}

As in the classical calculation of the normal lubrication force between spheres in a squeezing flow, the pressure gradient can be calculated by using the law of conservation of mass. As in the mono-viscous case, in the region $\mathcal{R}_{I}$ is given by ${ }^{19}$

$$
\left.\frac{\partial p}{\partial r}(r)\right|_{I}=-\frac{6 \eta_{0} r V}{h^{3}(r)}
$$

In the case of the region $\mathcal{R}_{I I}$, the conservation of mass reads

$$
\left.\pi r^{2} V\right|_{I I}=\left.2 \pi r \int_{z_{1}(r)}^{z_{2}(r)} u(r, z)\right|_{I I} d z
$$


The integration in the latter equation is done by using the expression of $\left.u(r, z)\right|_{I I}$ from Eq. (4), with the next result

$$
\left.\int_{z_{1}(r)}^{z_{2}(r)} u(r, z)\right|_{I I} d z=\frac{1}{12} \dot{\gamma}_{c} h^{2}(r)\left(3-\left(\frac{2 z_{c}(r)}{h(r)}\right)^{2}\right)
$$

where the relation between $z_{c}$ and $\partial p / \partial r$, Eq. (2), has been used. By replacing Eqs. (20) and (5) in (19), the next quadratic equation in $z_{c}$ is obtained

$$
\left(\frac{2 z_{c}(r)}{h(r)}\right)^{2}+2 \frac{\dot{\gamma}_{s}(r)}{\dot{\gamma}_{c}}-3=0
$$

which can be easily solved, obtaining

$$
\left.\frac{2 z_{c}(r)}{h(r)}\right|_{I I}=\sqrt{3-2 \frac{\dot{\gamma}_{s}(r)}{\dot{\gamma}_{c}}}
$$

Note that if $\dot{\gamma}_{s}(r) / \dot{\gamma}_{c}=1$ then $2 z_{c}(r) / h(r)=1$, ensuring the continuity of this solution between the regions $\mathcal{R}_{I}$ and $\mathcal{R}_{I I}$. It is also important to realize that when $\dot{\gamma}_{s}(r)>\frac{3}{2} \dot{\gamma}_{c}$ the solution is not real. That means that the mass conservation law can not be hold for any value of $z_{c}(r)$. However, this limit is never achieved because region III is reached beforehand. Indeed we can calculate what is the critical shear rate $\dot{\gamma}_{c}^{\prime}$, where, as it was said before, the $\mathcal{R}_{I I I}$ is not a null set anymore. This occurs when $2 z_{c}^{\prime}\left(r_{\max }\right) / h\left(r_{\max }\right)=1$. By applying Eqs. (22) and (2) to this condition one can conclude that $\dot{\gamma}_{c}^{\prime}$ is defined as follows

$$
\dot{\gamma}_{c}^{\prime}=\frac{1}{2}\left(3-\left(\frac{\eta_{0}}{\eta_{1}}\right)^{2}\right) \dot{\gamma}_{c}
$$

Note that the Eq. (22) for $\dot{\gamma}_{s}(r)=\dot{\gamma}_{c}^{\prime}$ is real, ensuring the real nature of such an equation for the whole region $\mathcal{R}_{I I}$. Note also that, as was explained in section II A, $\dot{\gamma}_{c}^{\prime}$ is not the real maximum shear rate that the biviscous fluid shows during the transition, but the maximum shear rate of the Newtonian reference.

According to Eq. (2), the pressure gradient can be obtained from Eq. (22), yielding

$$
\left.\frac{\partial p}{\partial r}\right|_{I I}=-\frac{2 \eta_{0} \dot{\gamma}_{c} h^{-1}(r)}{\sqrt{3-2 \frac{\dot{\gamma}_{s}(r)}{\dot{\gamma}_{c}}}}
$$

The same procedure can be followed to calculate the pressure gradient in the region $\mathcal{R}_{I I I}$. The conservation of mass applied to such a region reads

$$
\left.\pi r^{2} V\right|_{I I I}=\left.2 \pi r \int_{z_{1}(r)}^{z_{2}(r)} u(r, z)\right|_{I I I} d z
$$


The integral on the latter equation can be calculated, obtaining

$$
\left.\int_{z_{1}(r)}^{z_{2}(r)} u(r, z)\right|_{I I I} d z=\frac{h^{2}}{12} \dot{\gamma}_{c} \frac{\left(1-\frac{\eta_{0}^{2}}{\eta_{1}^{2}}\right)\left(\frac{2 z_{c}^{\prime}}{h}\right)^{3}+2}{\left(\frac{2 z_{c}^{\prime}}{h}\right)}
$$

where Eq. (2) has been used again. By replacing Eqs. (26) and (5) in (25), the next cubic equation in $z_{c}^{\prime}$ is obtained

$$
\left(1-\frac{\eta_{0}^{2}}{\eta_{1}^{2}}\right)\left(\frac{2 z_{c}^{\prime}}{h}\right)^{3}-2 \frac{\dot{\gamma}_{s}}{\dot{\gamma}_{c}}\left(\frac{2 z_{c}^{\prime}}{h}\right)+2=0
$$

One can check that if $2 z_{c}^{\prime} / h=1$ the shear rate $\dot{\gamma}_{s}(r)$ equals to $\dot{\gamma}_{c}^{\prime}$ as should be expected. Eq. (27) is a depressed cubic equation of the form $t^{3}+p t+q=0$, where

$$
\begin{aligned}
& q=2\left(\frac{\eta_{1}^{2}}{\eta_{1}^{2}-\eta_{0}^{2}}\right) \\
& p=-q \frac{\dot{\gamma}_{s}(r)}{\dot{\gamma}_{c}}
\end{aligned}
$$

It can be demonstrated that the solution to this equation is given by

$$
\begin{gathered}
\left.\frac{2 z_{c}^{\prime}}{h}\right|_{I I I}=-2 \sqrt{-\frac{p}{3}} \cos \left(\frac{\theta+4 \pi}{3}\right) \\
\cos \theta=-\frac{3 q}{2 p} \sqrt{-\frac{3}{p}}, \quad 0<\theta<\frac{\pi}{2}
\end{gathered}
$$

The pressure gradient $\partial p /\left.\partial r\right|_{I I I}$ can be calculated from $z_{c}^{\prime}$ by using Eq. (2).

In order to calculate the pressure $p(r)$ the pressure gradient must be integrated along the $r$ coordinate. We have not found any analytical solutions to the integrals of the exact pressure gradients in regions $\mathcal{R}_{I I}$ and $\mathcal{R}_{I I I}$, so in the next section we will describe how to obtain approximated analytical solutions.

\section{E. Pressure gradient approximations}

In order to derive a practical analytical solution, we propose an approximated analytical expression for the pressure gradient. Such an expression must allow to be analytically integrated twice in order to calculate the force. 
Case $\dot{\gamma}_{c}<\dot{\gamma}_{s}^{\max }<\dot{\gamma}_{c}^{\prime}$

The condition that the maximum shear rate $\dot{\gamma}_{s}^{\max }$ lies between $\dot{\gamma}_{c}$ and $\dot{\gamma}_{c}^{\prime}$ means that there is no coordinate $r \in \mathcal{R}_{I I I}$ As a consequence the region of coordinates $r$ in the regime II is given by $r_{1}<r \leq r_{2}$. In this case, the only continuity issues on the pressure gradient expression that we should consider are the transitions between regimes I and II (at $r_{1}$ and $r_{2}$ ). Specifically, we are going to approximate $2 z_{c}(r) /\left.h(r)\right|_{I I}$ given by Eq. (22). Such a function has a minimum between $r_{1}$ and $r_{2}$ at $r_{\max }=\sqrt{\frac{2}{3} a h_{0}}$, equal to $z^{\max }=2 z_{c}(r) /\left.h(r)\right|_{I I} ^{\max }=$ $\sqrt{3-27 r_{\max }|V| /\left(8 \dot{\gamma}_{c} h_{0}^{2}\right)}$, so one can approximate this function with a two-piecewise linear function. The first piece will interpolate this function at $r_{1}$ and $r_{\text {max }}$, and the second piece at $r_{\max }$ and $r_{2}$. The approximated pressure gradient reads then

$$
\left.\frac{\partial p}{\partial r}(r)\right|_{I I} \approx-2 \eta_{0} \dot{\gamma}_{c}\left[h(r)\left(m_{k} r+n_{k}\right)\right]^{-1}
$$

where

$$
\begin{array}{r}
m_{k}=-\frac{\left(1-z_{\max }\right)}{\left(r_{\max }-r_{k}\right)} \\
n_{k}=\frac{r_{\max }-r_{k} z_{\max }}{\left(r_{\max }-r_{k}\right)}
\end{array}
$$

and

$$
r_{k}= \begin{cases}r_{1}, & \text { if } r_{1}<r \leq r_{\max } \\ r_{2}, & \text { if } r_{\max }<r \leq r_{2}\end{cases}
$$

Case $\dot{\gamma}_{s}^{\max } \geq \dot{\gamma}_{c}^{\prime}$

When $\dot{\gamma}_{s}^{\max } \geq \dot{\gamma}_{c}^{\prime}$ there will be a non null region $r_{1}^{\prime}<r \leq r_{2}^{\prime}$ in the regime $I I I$, and two regions $r_{1}<r \leq r_{1}^{\prime}$ and $r_{2}^{\prime}<r \leq r_{2}^{\prime}$ in the regime $I I$. The pressure gradient approximation at $\mathcal{R}_{I I}$ will be done as it was in the previous section but interpolating at $r_{1}$ and $r_{1}^{\prime}$ for the first piece, and at $r_{2}^{\prime}$ and $r_{2}$ for the second one. The approximation of the pressure gradient at $\mathcal{R}_{I I}$ is given then by

where

$$
\left.\frac{\partial p}{\partial r}(r)\right|_{I I} \approx-2 \eta_{0} \dot{\gamma}_{c}\left[h(r)\left(m_{j k} r+n_{j k}\right)\right]^{-1}
$$

$$
\begin{aligned}
m_{j k} & =-\frac{1-\left(\eta_{0} / \eta_{1}\right)}{\left(r_{j}-r_{k}\right)} \\
n_{j k} & =\frac{r_{j}-r_{k}\left(\eta_{0} / \eta_{1}\right)}{\left(r_{j}-r_{k}\right)}
\end{aligned}
$$


and

$$
\begin{aligned}
& r_{k}= \begin{cases}r_{1}, & \text { if } r_{1}<r \leq r_{1}^{\prime} \\
r_{2}, & \text { if } r_{2}^{\prime}<r \leq r_{2}\end{cases} \\
& r_{j}= \begin{cases}r_{1}^{\prime}, & \text { if } r_{1}<r \leq r_{1}^{\prime} \\
r_{2}^{\prime}, & \text { if } r_{2}^{\prime}<r \leq r_{2}\end{cases}
\end{aligned}
$$

In general, we have checked that the pressure gradient in the region $\mathcal{R}_{I I I}$ can not be satisfactorily approximated by a one or two-piecewise linear function as was done for the region $\mathcal{R}_{I I}$. So, in order to find an approximation of $\partial p / \partial r$ at this region, a different route will be followed which is similar to the one used in Ref. ${ }^{19}$. If Eq. (27) is written in terms of $x=2 z_{c}^{\prime} / h$, the next cubic equation is obtained

$$
A(x)=x^{3}+p x+q=0
$$

where $p$ and $q$ have already been defined in Eq. (28). This function will be approached by its linear interpolation at points $x=0$ and $x=1$. Note that taking the interpolation point at $x=0$ ensures the continuity of this expression for the transition between $\mathcal{R}_{I I}$ and $\mathcal{R}_{I I I}$. The interpolation at $x=1$ ensures a good behavior of this approximation when the pressure gradient is very big and $2 z_{c}^{\prime} / h$ is close to 1 . With this interpolation, the approximated $A(x)$ function reads

$$
\left.A(x)\right|_{\text {approx }} \approx(1+p) x+q
$$

Introducing such an approximation at Eq. (37) the next expression for $z_{c}^{\prime}$ can be found

$$
\frac{2 z_{c}^{\prime}}{h} \approx-\frac{2}{1-2 \frac{\dot{\gamma}_{s}}{\dot{\gamma}_{c}}-\frac{\eta_{0}^{2}}{\eta_{1}^{2}}}
$$

and from the latter an approximation for the pressure gradient at $\mathcal{R}_{I I I}$ can be calculated

$$
\left.\frac{\partial p}{\partial r}(r)\right|_{I I I} \approx \eta_{1}\left(\frac{\dot{\gamma}_{c}}{h(r)}\left(1-\frac{\eta_{0}^{2}}{\eta_{1}^{2}}\right)-6 V \frac{r}{h^{3}(r)}\right)
$$

\section{LUBRICATION FORCE}

In order to calculate the force, the pressure gradient must be integrated first to obtain an expression for the pressure, as follows

$$
p(r)=-\int_{r}^{\infty} \frac{\partial p}{\partial r}(r) d r
$$


More details about the calculation of the pressure and the detailed results obtained can be consulted in the appendix A. From the pressure the lubrication force can be calculated by integration as

$$
F=\int_{0}^{\infty} p(r) 2 \pi r d r
$$

The reader is referred to the appendix B to consult the explicit calculation of the lubrication force. For the sake of clarity just the final result is shown here, which reads

$$
F=\int_{0}^{\infty} p(r) 2 \pi r d r= \begin{cases}F_{1}, & \text { if } h_{0} \geq h_{0}^{\text {lim }} \\ F_{2}, & \text { if } h_{0}^{\prime l i m} \leq h_{0}<h_{0}^{\text {lim }} \\ F_{3}, & \text { if } h_{0}<h_{0}^{\prime \text { lim }}\end{cases}
$$

with $h_{0}^{\text {lim }}$ and $h_{0}^{\prime l i m}$ defined in Eq.(16)-(17) and with the next expressions for $F_{1}, F_{2}$ and $F_{3}$

$$
\begin{aligned}
F_{1}= & 6 \pi \eta_{0} V a^{2} h_{0}^{-1} \\
F_{2}= & 6 \pi \eta_{0} V a^{2}\left(h_{0}^{-1}+h^{-1}\left(r_{2}\right)-h^{-1}\left(r_{1}\right)\right)+3 \pi \eta_{0} V a\left(\left(\frac{r_{2}}{h\left(r_{2}\right)}\right)^{2}-\left(\frac{r_{1}}{h\left(r_{1}\right)}\right)^{2}\right)+ \\
& 2 \pi \eta_{0} \dot{\gamma}_{c} a \lambda_{2} \\
F_{3}= & 6 \pi \eta_{0} V a^{2}\left(h_{0}^{-1}+h^{-1}\left(r_{2}\right)-h^{-1}\left(r_{1}\right)\right)+6 \pi \eta_{1} V a^{2}\left(h^{-1}\left(r_{1}^{\prime}\right)-h^{-1}\left(r_{2}^{\prime}\right)\right)+ \\
& 3 \pi \eta_{0} V a\left(\left(\frac{r_{2}}{h\left(r_{2}\right)}\right)^{2}-\left(\frac{r_{1}}{h\left(r_{1}\right)}\right)^{2}\right)-3 \pi \eta_{1} V a\left(\left(\frac{r_{2}^{\prime}}{h\left(r_{2}^{\prime}\right)}\right)^{2}-\left(\frac{r_{1}^{\prime}}{h\left(r_{1}^{\prime}\right)}\right)^{2}\right)+ \\
& 2 \pi \eta_{0} \dot{\gamma}_{c} a \lambda_{3}
\end{aligned}
$$

where $\lambda_{2}$ and $\lambda_{3}$ are defined in the Appendix.

\section{RESULTS}

In Fig. 3 we have compared the approximated analytical expression proposed in Eq. (44) with the exact solution calculated numerically for three different viscosity ratios $\eta_{1} / \eta_{0}=$ $10,100,1000$. The other parameters are the reduced particle radius $a=25 \mu \mathrm{m}$ corresponding to a typical non-colloidal case (particle radii $a_{1}=a_{2}$ ), low-viscosity plateau $\eta_{0}=10^{-3} \mathrm{~Pa} \cdot \mathrm{s}$, high-viscosity plateau is $\eta_{1}$. Finally, critical shear-rate for DST transition is chosen to be $\dot{\gamma}_{c}=6000 \mathrm{~s}^{-1}$ and the normally approaching particle velocity $V=0.1 \mathrm{~mm} / \mathrm{s}$. At 'large'

interparticle distances (still much smaller than $a$, to be in the lubrication regime), the hydrodynamic lubrication interaction between two spheres mediated by the DST solvent liquid 


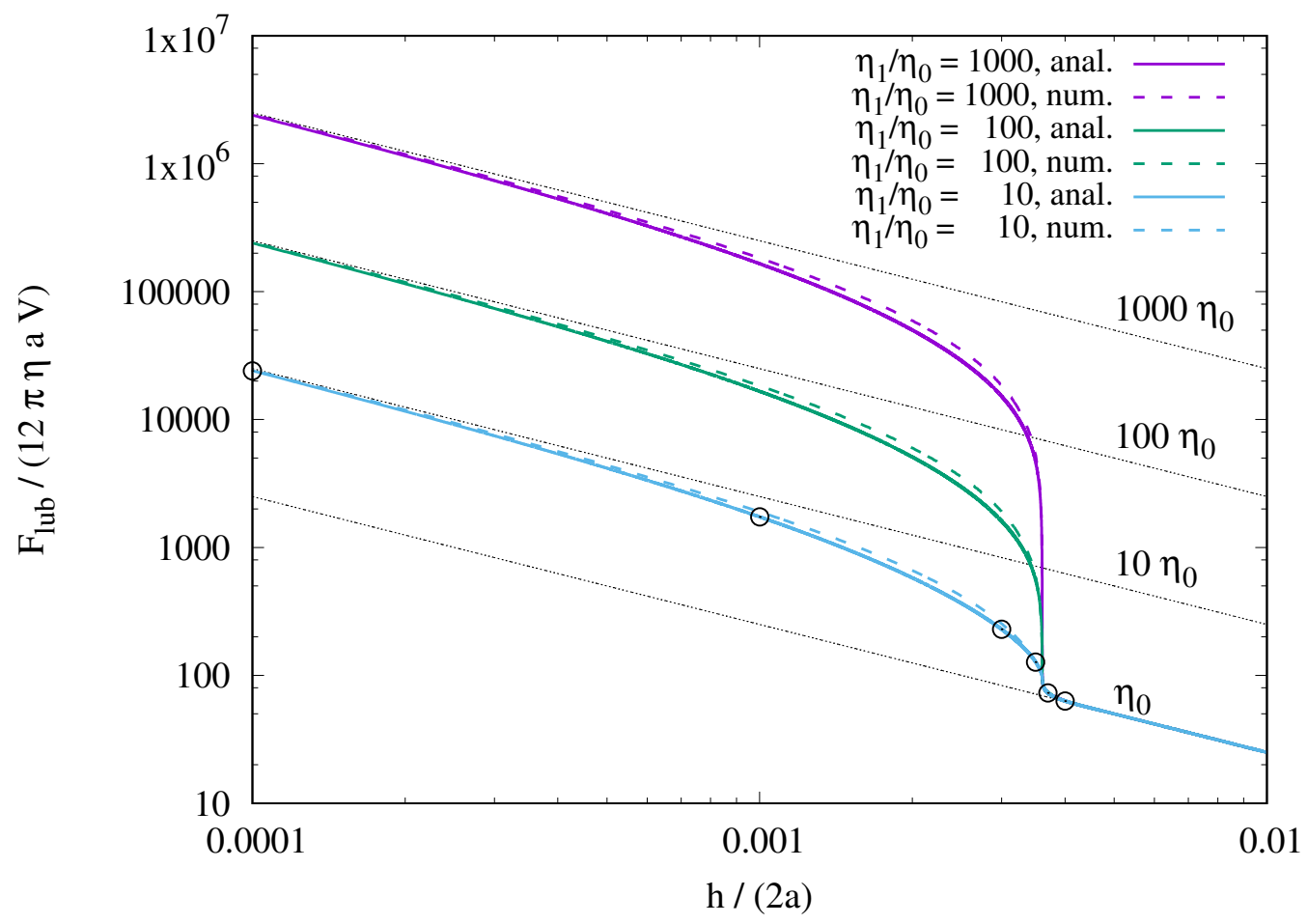

FIG. 3. Approximated analytical force (solid lines) compared to the numerical calculation (dashed lines) of the exact solution for different ratios $\eta_{1} / \eta_{0}$. The circles correspond to the frames shown at Fig. 4.

behaves as the expected Newtonian force with viscosity $\eta_{0}$. Similarly, at very small interparticle distances, the Newtonian lubrication force with viscosity $\eta_{1}$ is recovered. In the middle, a transition between the two regimes is observed which is in excellent agreement with the exact result estimated numerically over a wide range of viscosity ratios considered. Recent rheological studies of spherical and cubic noncolloidal particles suspended in model shear thickening colloidal fluids have been interpreted in terms of an effective, shear thickening suspending medium and provide motivation for developing accurate and robust descriptions of such systems ${ }^{25,28}$. Note also the similarity of the results of Fig. 3 with those of Potanin and Russel ${ }^{12}$ describing the hydrodynamic interactions of particles grafted with polymer brushes where the Brinkman's equations are used to solve the slow flow in the near-wall polymeric layer.

In order to explore in detail the flow features of the DST transition, in Fig. 4 we plot in the narrow gap between approaching spheres the different regions at viscosity, respectively, $\eta_{0}, \eta_{1}$ and transitional. The spheres shown in this figure are separated by different gaps 

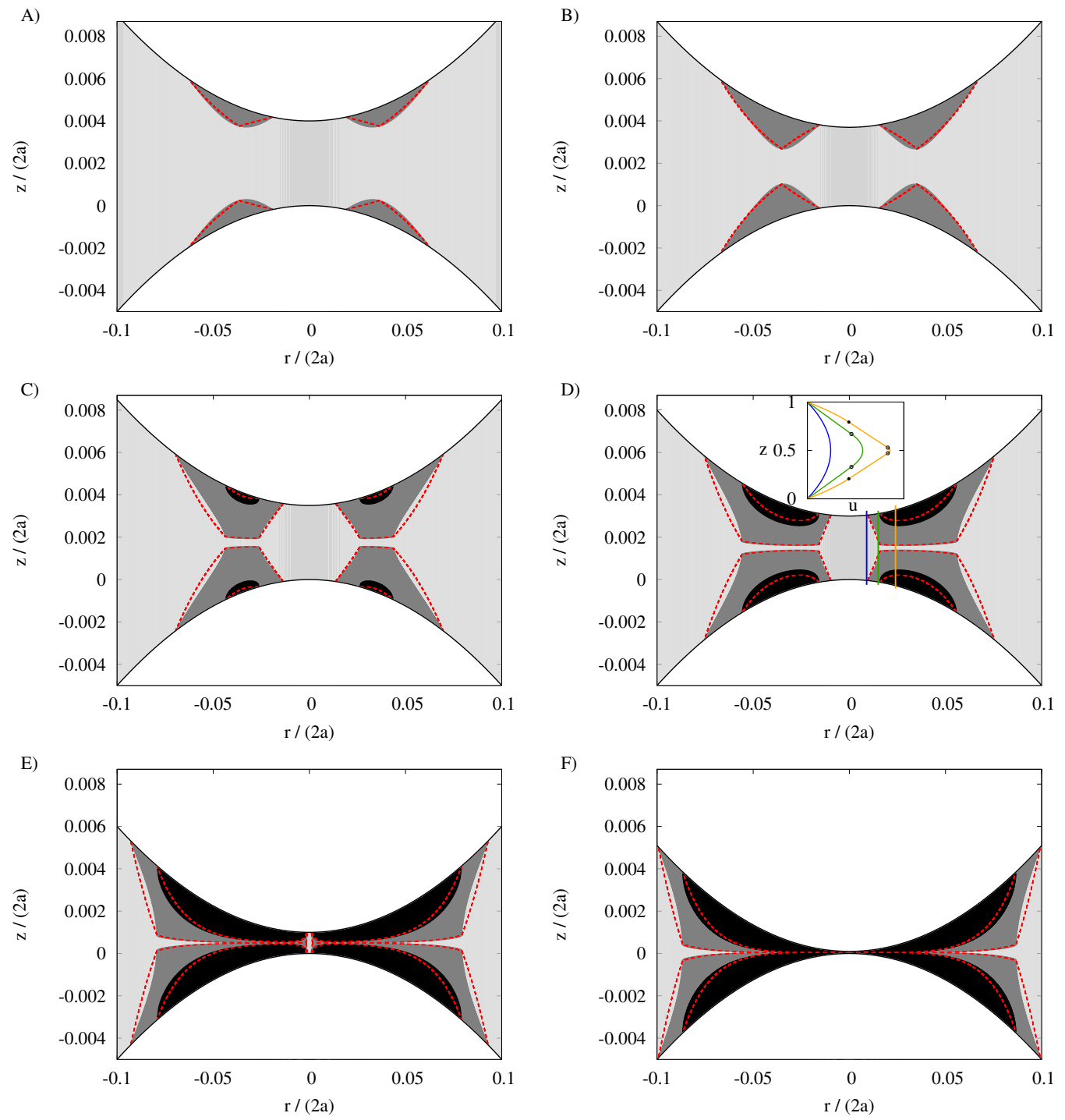

FIG. 4. Regions between two spheres with the same radius. The parameters are $a=25 \mu m$, $a_{1}=a_{2}, \eta_{0}=10^{-3} \mathrm{~Pa} \cdot \mathrm{s}, \eta_{1}=10 \eta_{0}, \dot{\gamma}_{c}=6000 \mathrm{~s}^{-1}$ and $V=0.1 \mathrm{~mm} / \mathrm{s}$ at distances $h_{0} /(2 a)=0.004$, 0.0037, 0.0035, 0.003, 0.001 and 0.0001. Spheres have been drawn with white color. Light gray, dark gray and black regions represent the exact analytical Newtonian region with $\eta_{0}$, the transitional region, and the Newtonian region with viscosity $\eta_{1}$ respectively. The analytical approximation used to calculate the lubrication force is depicted with red dashed lines. In the inset of plot (D) the squeezing flow profiles corresponding to three typical sections -drawn as vertical lines in the particle gap- are also shown. 

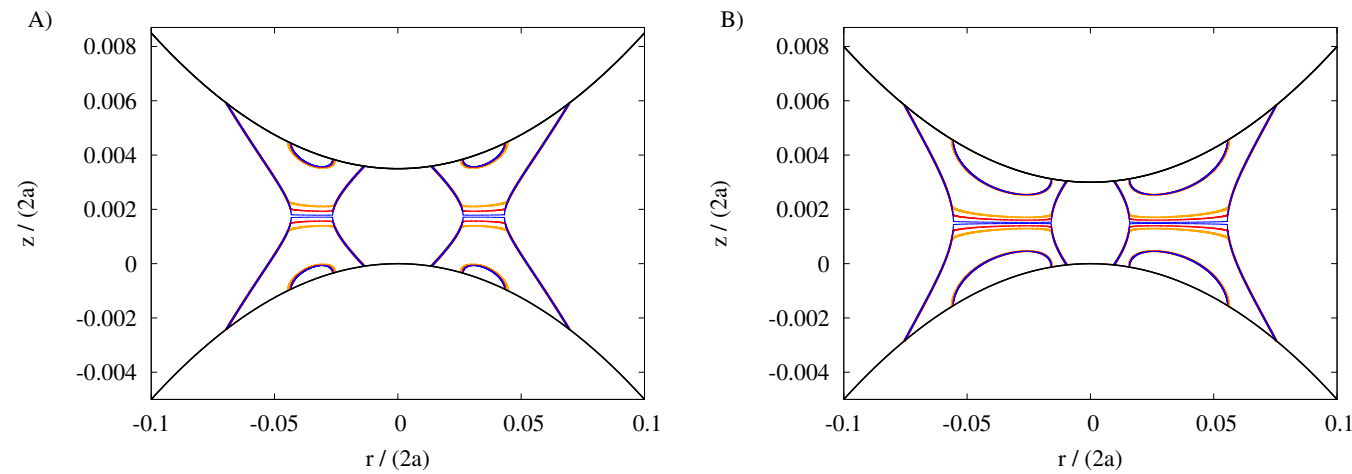

FIG. 5. Effect of viscosity ratio $\eta_{1} / \eta_{0}$ on three flow topology. Regions between two spheres with the same radius. The parameters are $a=25 \mu \mathrm{m}, a_{1}=a_{2}, \eta_{0}=10^{-3} \mathrm{~Pa} \cdot s, \dot{\gamma}_{c}=6000 \mathrm{~s}^{-1}$ and $V=0.1 \mathrm{~mm} / \mathrm{s}$ at distances $h_{0} /(2 a)=0.0035$ (left), and $h_{0} /(2 a)=0.003$ (right). Lines separating different regions are drawn for $\eta_{1} / \eta_{0}=5,10,50$ (orange,red and blue respectively).

corresponding to the circles drawn at Fig. 3. The exact analytical regions have been drawn as shaded regions, whereas the approximated analytical regions limits have been depicted by red dashed lines. In the inset the squeezing flow profiles corresponding to three typical sections -drawn as vertical lines in the particle gap- are shown. Blue, green and orange lines correspond to profiles in regions $\mathcal{R}_{I}, \mathcal{R}_{I I}$ and $\mathcal{R}_{I I I}$ respectively. The circles on the profiles indicate the position of $z=c \pm z_{c}$ and $z=c \pm z_{c}^{\prime}$.

As discussed above, at 'large' distances no transition is observed and the behavior is trivially Newtonian (regime I: not shown here). At moderate distances (Fig. 4 A), a first transition is triggered with the fluid behaving quasi-everywhere as Newtonian (light gray) except in two small areas (dark gray) close to the sphere's surfaces where the shear-rate is constant (regime II). These gray regions grow as particles get closer (Fig. 4 B) as a result of the increase of the region II, until a second transition (Fig. 4 C) occurs with two new areas (black) appearing again near the sphere's surfaces (regime III). The size of the areas progressively increases pervading the entire space in the gap, with the exception of a small region located symmetrically along the center-to-center distance (Fig. 4 D, E). Note also the thin horizontal region connecting the central region of viscosity $\eta_{0}$ with the outside flow domain (also at viscosity $\eta_{0}$ ). The connecting region becomes progressively thinner as the two spheres get closer until finally, for very small gaps (Fig. 4 F) the liquid within the gap is basically behaving as a Newtonian liquid with viscosity $\eta_{1}$. 
Finally, in Fig. 5 the effect of the viscosity ratio is shown. All the parameters are fixed as in Fig. 4 and the flow topology is shown for three different ratios $\left(\eta_{1} / \eta_{0}=1,10,50\right)$ corresponding to orange,red and blue lines respectively. The lines denote the transition between the different regimes in Fig. 4. Two different interparticle distances are considered: (A) $h_{0} / 2 a=0.0035$ and $(\mathrm{B}) h_{0} / 2 a=0.003$. It is clear that an increasing $\eta_{1} / \eta_{0}$ has the

effect to merge the two transitional regions (gray areas in Fig. 4) leaving a narrow thin area between them where the fluid did not reach the critical shear rate and it behaves as Newtonian with viscosity $\eta_{0}$.

A final remark on the validity of the DST lubrication model is in order. All the previous considerations are based on the premise to have a continuum-like behaviour of the suspending medium. In particular, if the suspending medium is a shear thickening colloidal dispersion such as those considered $\mathrm{in}^{25}$, the minimal scales should be $h_{0} \gg R_{\text {coll }}$ where $R_{\text {coll }}$ is the size of the small colloidal particle forming the dispersion. Typically a ratio $h_{0} / R_{\text {coll }} \sim 5-10$ is considered sufficient to have continuum-like behaviour on the scale $h_{0}$. On the other hand, in order to have a lubrication approximation between two spheres immersed in this medium, it is required that $h_{0} / a \ll 1$ where $a$ is the radius of the large non-colloidal particles (Fig. 1). Typically $h_{0} / a \lesssim 0.1$ is a reasonable choice. Therefore our lubrication model is accurate for dimensionless interparticle distances: $R_{\text {coll }} / a<h_{0} / a<0.1$ (Fig. 1). At larger distances $h_{0}$ lubrication does not hold. At smaller distances the continuum assumption breaks down. Therefore, the smaller the ratio $R_{\text {coll }} / a$, the wider will be the regime of applicability of the present model.

\section{CONCLUSIONS}

Based on the profile of a bi-viscous shear-thickening fluid in a Poiseuille flow calculated in Ref. ${ }^{26}$, an approximate analytical solution of the lubrication force between two spheres approaching/departing each other in a squeezing DST solvent medium is obtained. The approximation has been taken in the expression of the pressure gradient in such a way that it can be integrated twice in order to obtain an expression for the total force. Such an analytical solution ensures the correct behavior at both limits of close and far distances of interparticle surfaces, where the force is equal to the Newtonian lubrication force with $\eta_{1}$ and $\eta_{0}$ viscosities respectively. For intermediate gaps, the agreement with the numerical 
calculation of the exact solution is excellent. The Newtonian normal lubrication force is also recovered when $\eta_{1}=\eta_{0}$ extending consistently the scenario described in ${ }^{19}$ for the shear thinning fluids to the shear thickening regime.

The calculated lubrication force is identical to the Newtonian case of viscosity $\eta_{0}$ (lowviscosity regime) for distances $h>h_{0}^{l i m}$. When the particles get closer the viscosity increases

abruptly (solid-like regime) until for very small gaps $\left(h<<h_{0}^{l i m}\right)$ the Newtonian lubrication force of viscosity $\eta_{1}$ is the dominant interaction (high-viscosity regime). The explanation of such a behavior has been done in terms of the viscosity regions distribution in the gap between particles.

The theoretical results obtained in this work can allow in the future to perform simulations of dense suspensions with a discontinuous shear-thickening matrix based on effective lubrication dynamics numerical approaches ${ }^{8,10,11,29}$. Such simulations can be validated against experimental data ${ }^{25,28}$ and should prove valuable for efficient modeling of important industrial systems, such as concrete ${ }^{30}$.

\section{ACKNOWLEDGMENTS}

Computing resources offered by HPC Wales via the Project No. HPCWT050 (Multiscale particle simulation for complex fluids) are gratefully acknowledged. A.V-Q also thanks support from MINECO (Spain) under grants No. FIS2013-47350-C5-1-R and FIS201786007-C3-1-P.

\section{Appendix A: Calculation of the pressure}

The pressure can be obtained from the pressure gradient by doing the next integral

$$
p(r)=-\int_{r}^{\infty} \frac{\partial p}{\partial r}(r) d r
$$

Two cases will be distinguished: when $\dot{\gamma}_{c}<\dot{\gamma}_{s}^{\max }<\dot{\gamma}_{c}^{\prime}$ and when $\dot{\gamma}_{s}^{\max }>\dot{\gamma}_{c}^{\prime}$. 
1. Case $\dot{\gamma}_{c}<\dot{\gamma}_{s}^{\max }<\dot{\gamma}_{c}^{\prime}$

In this case the region $\mathcal{R}_{I I I}$ is null, and the pressure can be calculated as follows, depending on the value of $r$ :

$$
\begin{aligned}
\left.p(r)\right|_{r<r_{1}} & =-\left.\int_{r}^{r_{1}} \frac{\partial p}{\partial r}(r)\right|_{I} d r-\left.\int_{r_{1}}^{r_{2}} \frac{\partial p}{\partial r}(r)\right|_{I I} d r-\left.\int_{r_{2}}^{\infty} \frac{\partial p}{\partial r}(r)\right|_{I} d r \\
\left.p(r)\right|_{r_{1} \leq r \leq r_{2}} & =-\left.\int_{r}^{r_{2}} \frac{\partial p}{\partial r}(r)\right|_{I I} d r-\left.\int_{r_{2}}^{\infty} \frac{\partial p}{\partial r}(r)\right|_{I} d r \\
\left.p(r)\right|_{r>r_{2}} & =-\left.\int_{r_{2}}^{\infty} \frac{\partial p}{\partial r}(r)\right|_{I} d r=3 \eta_{0} V a h^{-2}(r)
\end{aligned}
$$

The explicit solution is

$$
\begin{aligned}
\left.p(r)\right|_{r \leq r_{1}}= & 3 \eta_{0} V a\left(h^{-2}(r)+h^{-2}\left(r_{2}\right)-h^{-2}\left(r_{1}\right)\right)+ \\
& 2 \eta_{0} \dot{\gamma}_{c} a\left(\phi\left(r_{1}, r_{\text {max }}, m_{1}, n_{1}\right)-\phi\left(r_{2}, r_{\text {max }}, m_{2}, n_{2}\right)\right) \\
\left.p(r)\right|_{r_{1}<r \leq r_{\max }}= & 3 \eta_{0} \operatorname{Vah}^{-2}\left(r_{2}\right)+2 \eta_{0} \dot{\gamma}_{c} a\left(\phi\left(r, r_{\max }, m_{1}, n_{1}\right)-\phi\left(r_{2}, r_{\max }, m_{2}, n_{2}\right)\right) \\
\left.p(r)\right|_{r_{\max }<r \leq r_{2}}= & 3 \eta_{0} \operatorname{Vah}^{-2}\left(r_{2}\right)-2 \eta_{0} \dot{\gamma}_{c} a \phi\left(r_{2}, r, m_{2}, n_{2}\right) \\
\left.p(r)\right|_{r>r_{2}}= & 3 \eta_{0} \operatorname{Vah}^{-2}(r)
\end{aligned}
$$

where

$$
\begin{aligned}
\phi\left(r_{a}, r_{b}, m, n\right)= & \frac{1}{3 r_{\text {max }}^{2} m^{2}+n^{2}}\left[\frac{2}{\sqrt{3}} \frac{n}{r_{\max }}\left(\arctan \left(\frac{1}{\sqrt{3}} \frac{r_{b}}{r_{\max }}\right)-\arctan \left(\frac{1}{\sqrt{3}} \frac{r_{a}}{r_{\max }}\right)\right)\right. \\
& \left.+m \ln \left(\left(\frac{m r_{b}+n}{m r_{a}+n}\right)^{2} \frac{h\left(r_{a}\right)}{h\left(r_{b}\right)}\right)\right]
\end{aligned}
$$


2. Case $\dot{\gamma}_{s}^{\max }>\dot{\gamma}_{c}^{\prime}$

In this case the region $\mathcal{R}_{I I I}$ is not null and the pressure can be calculated as follows depending on the value of $r$ :

$$
\begin{aligned}
\left.p(r)\right|_{r<r_{1}}= & -\left.\int_{r}^{r_{1}} \frac{\partial p}{\partial r}(r)\right|_{I} d r-\left.\int_{r_{1}}^{r_{1}^{\prime}} \frac{\partial p}{\partial r}(r)\right|_{I I} d r-\left.\int_{r_{1}^{\prime}}^{r_{2}^{\prime}} \frac{\partial p}{\partial r}(r)\right|_{I I I} d r- \\
& \left.\int_{r_{2}^{\prime}}^{r_{2}} \frac{\partial p}{\partial r}(r)\right|_{I I} d r-\left.\int_{r_{2}}^{\infty} \frac{\partial p}{\partial r}(r)\right|_{I} d r \\
\left.p(r)\right|_{r_{1} \leq r \leq r_{1}^{\prime}}= & -\left.\int_{r}^{r_{1}^{\prime}} \frac{\partial p}{\partial r}(r)\right|_{I I} d r-\left.\int_{r_{1}^{\prime}}^{r_{2}^{\prime}} \frac{\partial p}{\partial r}(r)\right|_{I I I} d r- \\
& \left.\int_{r_{2}^{\prime}}^{r_{2}} \frac{\partial p}{\partial r}(r)\right|_{I I} d r-\left.\int_{r_{2}}^{\infty} \frac{\partial p}{\partial r}(r)\right|_{I} d r \\
\left.p(r)\right|_{r_{1}^{\prime} \leq r \leq r_{2}^{\prime}}= & -\left.\int_{r}^{r_{2}^{\prime}} \frac{\partial p}{\partial r}(r)\right|_{I I I} d r-\left.\int_{r_{2}^{\prime}}^{r_{2}} \frac{\partial p}{\partial r}(r)\right|_{I I} d r-\left.\int_{r_{2}}^{\infty} \frac{\partial p}{\partial r}(r)\right|_{I} d r \\
\left.p(r)\right|_{r_{1}^{\prime} \leq r \leq r_{2}^{\prime}}= & -\left.\int_{r}^{r_{2}} \frac{\partial p}{\partial r}(r)\right|_{I I} d r-\left.\int_{r_{2}}^{\infty} \frac{\partial p}{\partial r}(r)\right|_{I} d r \\
\left.p(r)\right|_{r>r_{2}}= & -\left.\int_{r_{2}}^{\infty} \frac{\partial p}{\partial r}(r)\right|_{I} d r=3 \eta_{0} V a h^{-2}(r)
\end{aligned}
$$

The explicit solution reads

$$
\begin{array}{rl}
\left.p(r)\right|_{r \leq r_{1}}= & 3 \eta_{0} V a\left(h^{-2}(r)+h^{-2}\left(r_{2}\right)-h^{-2}\left(r_{1}\right)\right)+3 \eta_{1} V a\left(h^{-2}\left(r_{1}^{\prime}\right)-h^{-2}\left(r_{2}^{\prime}\right)\right)+ \\
& 2 \eta_{0} \dot{\gamma}_{c} a\left(\phi\left(r_{1}, r_{1}^{\prime}, m_{1^{\prime} 1}, n_{1^{\prime} 1}\right)-\phi\left(r_{2}, r_{2}^{\prime}, m_{2^{\prime} 2}, n_{2^{\prime} 2}\right)\right)- \\
& \left(\frac{\eta_{1}^{2}-\eta_{0}^{2}}{\eta_{1}}\right) \dot{\gamma}_{c} \alpha\left(r_{2}^{\prime}, r_{1}^{\prime}\right) \\
\left.p(r)\right|_{r_{1}<r \leq r_{1}^{\prime}}= & 3 \eta_{0} V a h^{-2}\left(r_{2}\right)+3 \eta_{1} V a\left(h^{-2}\left(r_{1}^{\prime}\right)-h^{-2}\left(r_{2}^{\prime}\right)\right)+ \\
2 & 2 \eta_{0} \dot{\gamma}_{c} a\left(\phi\left(r, r_{1}^{\prime}, m_{1^{\prime} 1}, n_{1^{\prime} 1}\right)-\phi\left(r_{2}, r_{2}^{\prime}, m_{2^{\prime} 2}, n_{2^{\prime} 2}\right)\right)- \\
& \left(\frac{\eta_{1}^{2}-\eta_{0}^{2}}{\eta_{1}}\right) \dot{\gamma}_{c} \alpha\left(r_{2}^{\prime}, r_{1}^{\prime}\right) \\
\left.p(r)\right|_{r_{1}^{\prime}<r \leq r_{2}^{\prime}}=3 \eta_{0} V a h^{-2}\left(r_{2}\right)+3 \eta_{1} V a\left(h^{-2}(r)-h^{-2}\left(r_{2}^{\prime}\right)\right)- \\
2 \eta_{0} \dot{\gamma}_{c} a \phi\left(r_{2}, r_{2}^{\prime}, m_{2^{\prime} 2}, n_{2^{\prime} 2}\right)- \\
\left(\frac{\eta_{1}^{2}-\eta_{0}^{2}}{\eta_{1}}\right) \dot{\gamma}_{c} \alpha\left(r_{2}^{\prime}, r\right) \\
\left.p(r)\right|_{r_{2}^{\prime}<r \leq r_{2}}=3 \eta_{0} V a h^{-2}\left(r_{2}\right)-2 \eta_{0} \dot{\gamma}_{c} a \phi\left(r_{2}, r, m_{2^{\prime} 2}, n_{2^{\prime} 2}\right) \\
\left.p(r)\right|_{r>r_{2}^{\prime}}=3 \eta_{0} V a h^{-2}(r)
\end{array}
$$

where the dimensionless function $\alpha$ is given by

$$
\alpha\left(r_{a}, r_{b}\right)=\sqrt{\frac{2 a}{h_{0}}}\left(\arctan \left(\frac{1}{\sqrt{3}} \frac{r_{b}}{r_{\max }}\right)-\arctan \left(\frac{1}{\sqrt{3}} \frac{r_{a}}{r_{\max }}\right)\right)
$$




\section{Appendix B: Calculation of the Force}

The lubrication force is calculated as follows

$$
F=\int_{0}^{\infty} p(r) 2 \pi r d r=\left\{\begin{array}{c}
F_{1}, \quad \text { if } h_{0} \geq h_{0}^{\text {lim }} \\
F_{2}, \quad \text { if } h_{0}^{\text {llim }} \leq h_{0}<h_{0}^{l i m} \\
F_{3}, \quad \text { if } h_{0}<h_{0}^{\prime l i m}
\end{array}\right.
$$

where $F_{1}, F_{2}$ and $F_{3}$ are calculated as follows

$$
\begin{aligned}
F_{1}= & \left.\int_{0}^{\infty} p(r)\right|_{\text {newt }} 2 \pi r d r \\
F_{2}= & \left.\int_{0}^{r_{1}} p(r)\right|_{r<r_{1}} 2 \pi r d r+\left.\int_{r_{1}}^{r_{2}} p(r)\right|_{r_{1} \leq r<r_{2}} 2 \pi r d r+\left.\int_{r_{2}}^{\infty} p(r)\right|_{r \geq r_{2}} 2 \pi r d r \\
F_{3}= & \left.\int_{0}^{r_{1}} p(r)\right|_{r<r_{1}} 2 \pi r d r+\left.\int_{r_{1}}^{r_{1}^{\prime}} p(r)\right|_{r_{1} \leq r<r_{1}^{\prime}} 2 \pi r d r+\left.\int_{r_{1}^{\prime}}^{r_{2}^{\prime}} p(r)\right|_{r_{1}^{\prime} \leq r<r_{2}^{\prime}} 2 \pi r d r+ \\
& \left.\int_{r_{2}^{\prime}}^{r_{2}} p(r)\right|_{r_{2}^{\prime} \leq r<r_{2}} 2 \pi r d r+\left.\int_{r_{2}}^{\infty} p(r)\right|_{r \geq r_{2}} 2 \pi r d r
\end{aligned}
$$

and they read explicitly

$$
\begin{aligned}
F_{1}= & 6 \pi \eta_{0} V a^{2} h_{0}^{-1} \\
F_{2}= & 6 \pi \eta_{0} V a^{2}\left(h_{0}^{-1}+h^{-1}\left(r_{2}\right)-h^{-1}\left(r_{1}\right)\right)+ \\
& 3 \pi \eta_{0} V a\left(\left(\frac{r_{2}}{h\left(r_{2}\right)}\right)^{2}-\left(\frac{r_{1}}{h\left(r_{1}\right)}\right)^{2}\right)+ \\
& 2 \pi \eta_{0} \dot{\gamma}_{c} a \lambda_{2} \\
F_{3}= & 6 \pi \eta_{0} V a^{2}\left(h_{0}^{-1}+h^{-1}\left(r_{2}\right)-h^{-1}\left(r_{1}\right)\right)+6 \pi \eta_{1} V a^{2}\left(h^{-1}\left(r_{1}^{\prime}\right)-h^{-1}\left(r_{2}^{\prime}\right)\right)+ \\
& 3 \pi \eta_{0} V a\left(\left(\frac{r_{2}}{h\left(r_{2}\right)}\right)^{2}-\left(\frac{r_{1}}{h\left(r_{1}\right)}\right)^{2}\right)-3 \pi \eta_{1} V a\left(\left(\frac{r_{2}^{\prime}}{h\left(r_{2}^{\prime}\right)}\right)^{2}-\left(\frac{r_{1}^{\prime}}{h\left(r_{1}^{\prime}\right)}\right)^{2}\right)+ \\
& 2 \pi \eta_{0} \dot{\gamma}_{c} a \lambda_{3}
\end{aligned}
$$


where

$$
\begin{aligned}
\lambda_{2}= & {\left[r_{1}^{2} \phi\left(r_{1}, r_{\max }, m_{1}, n_{1}\right)-r_{\max }^{2} \phi\left(r_{2}, r_{\max }, m_{2}, n_{2}\right)+\right.} \\
& \sigma\left(r_{\max }, r_{\max }, m_{1}, n_{1}\right)-\sigma\left(r_{1}, r_{\max }, m_{1}, n_{1}\right)+ \\
& \left.\sigma\left(r_{2}, r_{2}, m_{2}, n_{2}\right)-\sigma\left(r_{\max }, r_{2}, m_{2}, n_{2}\right)\right] \\
\lambda_{3}= & {\left[\left(\frac{\eta_{1}}{\eta_{0}}-\frac{\eta_{0}}{\eta_{1}}\right)\left(r_{1}^{\prime}-r_{2}^{\prime}\right)+r_{1}^{2} \phi\left(r_{1}, r_{1}^{\prime}, m_{1^{\prime} 1}, n_{1^{\prime} 1}\right)-r_{2}^{\prime 2} \phi\left(r_{2}, r_{2}^{\prime}, m_{2^{\prime} 2}, n_{2^{\prime} 2}\right)+\right.} \\
& \sigma\left(r_{1}^{\prime}, r_{1}^{\prime}, m_{1^{\prime} 1}, n_{1^{\prime} 1}\right)-\sigma\left(r_{1}, r_{1}^{\prime}, m_{1^{\prime} 1}, n_{1^{\prime} 1}\right)+ \\
& \left.\sigma\left(r_{2}, r_{2}, m_{2^{\prime} 2}, n_{2^{\prime} 2}\right)-\sigma\left(r_{2}^{\prime}, r_{2}^{\prime}, m_{2^{\prime} 2}, n_{2^{\prime} 2}\right)\right]+ \\
& \pi \sqrt{\frac{2 a}{h_{0}}} \dot{\gamma}_{c} \frac{\left(\eta_{1}^{2}-\eta_{0}^{2}\right)}{\eta_{1}} \times \\
& \left(\left(r_{1}^{\prime 2}-2 a h\left(r_{1}^{\prime}\right)\right) \arctan \left(\frac{1}{\sqrt{3}} \frac{r_{1}^{\prime}}{r_{\max }}\right)-\left(r_{2}^{\prime 2}-2 a h\left(r_{2}^{\prime}\right)\right) \arctan \left(\frac{1}{\sqrt{3}} \frac{r_{2}^{\prime}}{r_{\max }}\right)\right)
\end{aligned}
$$

being

$$
\begin{aligned}
\sigma\left(r_{a}, r_{b}, m, n\right) \equiv & 2 \int \phi\left(r_{a}, r_{b}, m, n\right) r_{a} d r_{a}=\frac{1}{3 m r_{\max }\left(n^{2}+3 m^{2} r_{\max }^{2}\right)} \times \\
& {\left[2 \sqrt{3} m n\left(-\left(3 r_{\text {max }}^{2}+r_{a}^{2}\right) \arctan \left(\frac{1}{\sqrt{3}} \frac{r_{a}}{r_{\max }}\right)+r_{a}^{2} \arctan \left(\frac{1}{\sqrt{3}} \frac{r_{b}}{r_{\max }}\right)\right)+\right.} \\
& \left.3 r_{\max }\left(2\left(n^{2}-m^{2} r_{a}^{2}\right) \ln \left(n+m r_{a}\right)+2 m^{2} a h\left(r_{a}\right) \ln \left(2 a h\left(r_{a}\right)\right)+m^{2} r_{a}^{2} \ln \left(\frac{\left(n+m r_{b}\right)^{2}}{2 a h\left(r_{b}\right)}\right)\right)\right]
\end{aligned}
$$

\section{REFERENCES}

${ }^{1}$ M. Maxey, "Simulation methods for particulate flows and concentrated suspensions," Annual Review of Fluid Mechanics 49, 171-193 (2017).

${ }^{2}$ Y. J. Choi, M. A. Hulsen, and H. E. Meijer, "An extended finite element method for the simulation of particulate viscoelastic flows," Journal of Non-Newtonian Fluid Mechanics 165, $607-624(2010)$.

${ }^{3}$ N. Jaensson, M. Hulsen, and P. Anderson, "Simulations of the start-up of shear flow of 2d particle suspensions in viscoelastic fluids: Structure formation and rheology," Journal of Non-Newtonian Fluid Mechanics 225, 70 - 85 (2015).

${ }^{4}$ G. D'Avino, F. Greco, M. A. Hulsen, and P. L. Maffettone, "Rheology of viscoelastic suspensions of spheres under small and large amplitude oscillatory shear by numerical simulations," Journal of Rheology 57, 813-839 (2013). 
${ }^{5}$ S. Krishnan, E. S. Shaqfeh, and G. Iaccarino, "Fully resolved viscoelastic particulate simulations using unstructured grids," Journal of Computational Physics 338, 313 - 338 (2017).

${ }^{6}$ N. S. Martys, W. L. George, B.-W. Chun, and D. Lootens, "A smoothed particle hydrodynamics-based fluid model with a spatially dependent viscosity: application to flow of a suspension with a non-newtonian fluid matrix," Rheologica Acta 49, 1059-1069 (2010).

${ }^{7}$ A. Vázquez-Quesada, R. I. Tanner, and M. Ellero, "Shear thinning of noncolloidal suspensions," Phys. Rev. Lett. 117, 108001 (2016).

${ }^{8}$ A. Vázquez-Quesada and M. Ellero, "Sph modeling and simulation of spherical particles interacting in a viscoelastic matrix," Physics of Fluids 29, 121609 (2017).

${ }^{9}$ Y. K. Lee and K. H. Ahn, "A novel lattice boltzmann method for the dynamics of rigid particles suspended in a viscoelastic medium," Journal of Non-Newtonian Fluid Mechanics 244, 75 - 84 (2017).

${ }^{10} \mathrm{X}$. Bian and M. Ellero, "A splitting integration scheme for the sph simulation of concentrated particle suspensions," Computer Physics Communications 185, 53-62 (2014).

${ }^{11}$ A. Vázquez-Quesada and M. Ellero, "Rheology and microstructure of non-colloidal suspensions under shear studied with smoothed particle hydrodynamics," Journal of NonNewtonian Fluid Mechanics (2016).

${ }^{12}$ A. A. Potanin and W. B. Russel, "Hydrodynamic interaction of particles with grafted polymer brushes and applications to rheology of colloidal dispersions," Phys. Rev. E 52, 730-737 (1995).

${ }^{13}$ G. Lian, Y. Xu, W. Huang, and M. J. Adams, "On the squeeze flow of a power-law fluid between rigid spheres," Journal of Non-Newtonian Fluid Mechanics 100, 151-164 (2001).

${ }^{14}$ A. R. Koblitz, S. Lovett, and N. Nikiforakis, "Viscoplastic squeeze flow between two identical infinite circular cylinders," Phys. Rev. Fluids 3, 023301 (2018).

${ }^{15}$ J. Mewis and N. J. Wagner, Colloidal Suspension Rheology (Cambridge University Press, 2011) cambridge Books Online.

${ }^{16}$ A. B. Metzner, "Rheology of suspensions in polymeric liquids," Journal of Rheology 29, 739-775 (1985).

${ }^{17}$ M. Liard, N. S. Martys, W. L. George, D. Lootens, and P. Hebraud, "Scaling laws for the flow of generalized newtonian suspensions," Journal of Rheology 58, 1993-2015 (2014). 
${ }^{18}$ A. Vázquez-Quesada, A. Mahmud, S. Dai, M. Ellero, and R. I. Tanner, "Investigating the causes of shear-thinning in non-colloidal suspensions: Experiments and simulations," Journal of Non-Newtonian Fluid Mechanics 248, 1 - 7 (2017).

${ }^{19}$ A. Vázquez-Quesada and M. Ellero, "Analytical solution for the lubrication force between two spheres in a bi-viscous fluid," Physics of Fluids 28, 073101 (2016).

${ }^{20}$ D. K. Gartling and N. Phan-Thien, "A numerical simulation of a plastic fluid in a parallelplate plastometer," Journal of non-Newtonian fluid mechanics 14, 347-360 (1984).

${ }^{21}$ A. Vázquez-Quesada, P. Español, and M. Ellero, "Apparent slip mechanism between two spheres based on solvent rheology: theory and implication on the shear-thinning of non-brownian suspensions," Physical Review Fluids under review (2018).

${ }^{22}$ N. J. Wagner and J. F. Brady, "Shear thickening in colloidal dispersions," Physics Today 62, 27-32 (2009).

${ }^{23}$ Y. S. Lee, E. D. Wetzel, and N. J. Wagner, "The ballistic impact characteristics of kevlarß) woven fabrics impregnated with a colloidal shear thickening fluid," Journal of materials science 38, 2825-2833 (2003).

${ }^{24}$ J. N. Fowler, A. A. Pallanta, C. B. Swanik, and N. J. Wagner, "The use of shear thickening nanocomposites in impact resistant materials," Journal of biomechanical engineering 137, 054504 (2015).

${ }^{25}$ C. D. Cwalina and N. J. Wagner, "Rheology of non-brownian particles suspended in concentrated colloidal dispersions at low particle reynolds number," Journal of Rheology 60, 47-59 (2016).

${ }^{26}$ A. Vázquez-Quesada, N. Wagner, and M. Ellero, "Planar channel flow of a discontinuous shear-thickening model fluid: Theory and simulation," Physics of Fluids 29, 103104 (2017).

${ }^{27}$ E. Guazzelli and J. F. Morris, A physical introduction to suspension dynamics (Cambridge University Press, 2011).

${ }^{28}$ C. D. Cwalina, K. J. Harrison, and N. J. Wagner, "Rheology of cubic particles suspended in a newtonian fluid," Soft Matter 12, 4654-4665 (2016).

${ }^{29}$ A. Vázquez-Quesada, X. Bian, and M. Ellero, "Three-dimensional simulations of dilute and concentrated suspensions using smoothed particle hydrodynamics," Computational Particle Mechanics 1, 36 (2015).

${ }^{30}$ F. Toussaint, C. Roy, and P.-H. Jézéquel, "Reducing shear thickening of cement-based suspensions," Rheologica Acta 48, 883-895 (2009). 
